\title{
Intervenções psicológicas com universitários em serviços de apoio ao estudante ${ }^{1}$
}

\author{
Fabiana Pinheiro Ramos ${ }^{2}$ \\ Alexsandro Luiz De Andrade \\ Adriano Pereira Jardim \\ Juliana Nascimento Lucas Ramalhete \\ Gustavo Pfister Pirola \\ Caroline Egert
}

Universidade Federal do Espírito Santo, Vitória-ES, Brasil

\section{Resumo}

O ingresso no ensino superior apresenta desafios, sendo importante oferecer programas que promovam a saúde mental do universitário e o preparem para a realidade profissional e para os desafios da carreira. Neste artigo são descritas propostas de intervenções psicológicas com universitários (objetivos e metodologias) desenvolvidas nos anos de 2015 a 2017, por 86 graduandos em Psicologia, e que beneficiaram 705 universitários: 1. acolhimento e triagem psicológica; 2 . oficinas: de preparação para a vida acadêmica, de habilidades sociais, de controle da ansiedade e enfrentamento do estresse, de orientação aos estudos, e de temática específica; 3. psicoterapia individual; e 4. educação para a carreira. Discute-se a contribuição dessas intervenções para a proposição de outros serviços que atendam às demandas da população universitária.

Palavras-chave: Ensino superior, ambiente universitário, intervenção psicológica, desenvolvimento profissional

\section{Abstract: Psychological interventions with university students in student support services}

Psychological interventions with university students in student support services

Admission to higher education presents challenges, and it is important to offer programs that promote the mental health of the university and prepare it for professional reality and for career challenges. In this article, psychological interventions with university students (objectives and methodologies) developed in the years 2015 to 2017 by 86 undergraduates in Psychology are described, benefiting 705 graduate students: 1. reception and psychological screening; 2. workshops: preparation for academic life, social skills, anxiety control and coping with stress, orientation to studies, and specific topics; 3 . individual psychotherapy; and 4. career education. We discuss the contribution of these interventions to the proposal of other services that meet the demands of the university population.

Keywords: Higher education, college environment, psychological intervention, professional development

\section{Resumen: Intervenciones psicológicas con universitarios en servicios de apoyo al estudiante}

El ingreso en la enseñanza superior presenta desafíos, siendo importante ofrecer programas que promuevan la salud mental del universitario y lo preparen para la realidad profesional y de la carrera. En este artículo se describen propuestas de intervenciones psicológicas con estudiantes universitarios (objetivos y metodologías) desarrolladas entre 2015 y 2017, por 86 graduandos en Psicología, y que beneficiaron a 705 estudiantes universitarios: 1. acogida y clasificación psicológica; 2. talleres: preparación para la vida académica, habilidades sociales, control de la ansiedad y enfrentamiento del estrés, orientación a los estudios, y de temática específica; 3. psicoterapia individual; y 4.educación para la carrera. Se discute la contribución de esas intervenciones para la proposición de otros servicios que atiendan a las demandas de la población universitaria.

Palabras clave: Educación superior, ambiente universitário, intervención psicológica, desarrollo professional

\footnotetext{
${ }^{1}$ Agradecemos a todos os graduandos em Psicologia que atuaram nas atividades descritas no artigo, à Pró-Reitoria de Planejamento e Desenvolvimento Institucional da Universidade Federal do Espírito Santo (UFES) pela concessão de bolsa para a 4ª autora, à Pró-Reitoria de Extensão da UFES pela concessão de bolsa para o $5^{\circ}$ autor, e à Pró-Reitoria de Graduação pela concessão de bolsa à $6^{\mathrm{a}}$ autora e aos demais integrantes do Projeto "Sou universitário e agora: uma preparação psicológica para a vida acadêmica".

${ }^{2}$ Endereço para correspondência: Departamento de Psicologia. Universidade Federal do Espírito Santo. Avenida Fernando Ferrari, 514, Goiabeiras, 29075-910, Vitória, ES. E-mail: fabiana.ramos@ufes.br
} 
A literatura evidencia que a transição do ensino médio para o ensino superior é complexa e pode envolver importantes desafios para aqueles que iniciam a vida universitária, além de exigir do estudante inúmeras habilidades para lidar com tais desafios e ser bem sucedido no ambiente acadêmico (Soares et al., 2014). A vivência do período de adaptação acadêmica pode ser considerada como preditora do sucesso e da satisfação no desenvolvimento da carreira profissional (Teixeira \& Costa, 2017).

A adaptação ao novo contexto costuma ser desafiadora para a maioria dos estudantes em seu período inicial na trajetória acadêmica, pois a maioria deles enfrenta tarefas complexas em âmbito acadêmico (novos ritmos e estratégias de aprendizagem, sistemas de ensino e avaliação), social (novos padrões de relacionamento, além da ampliação da rede social), pessoal (estabelecimento de um sentido mais forte de identidade) e vocacional, com a definição de metas de carreira (Moreno \& Soares, 2014). A não adaptação ao contexto acadêmico pode contribuir para a retenção e evasão escolares (Ambiel, Santos, \& Dalbosco, 2016).

Torna-se importante, portanto, compreender as principais dificuldades vivenciadas pelos estudantes durante esse período, no exercício do seu novo papel social, o de acadêmico universitário, sobretudo para prevenir aquelas situações que podem contribuir para o adoecimento do estudante ou para o agravamento de quadros pré-existentes à entrada na universidade, conforme demonstra a literatura da área (Friedrich, Macedo, \& Reis, 2015; Padovani et al., 2014). Nesse campo de investigação, o do sofrimento e adoecimento psicológico em acadêmicos, destacam-se também os trabalhos que apontam para o uso abusivo de drogas lícitas e ilícitas entre a população universitária, como forma de enfrentar os desafios vivenciados no ensino superior (Eckschmidt, Andrade, \& Oliveira, 2013; Zeferino et al., 2015). Assim, são necessários programas de intervenção que possam oferecer suporte a essa população, mediante oferta de serviços psicológicos voltados para o desenvolvimento de habilidades específicas requeridas na vida profissional e pessoal do acadêmico, diminuindo fatores de risco para o adoecimento e contribuindo para sua saúde mental (Ferreira, Oliveira, \& Vandenberg, 2014; Lantyer, Varanda, Souza, Padovani, \& Viana, 2016; Silva \& Tucci, 2015).

A literatura demonstra que o atendimento às necessidades psicológicas de universitários por meio de propostas de intervenção ainda é escasso (Amaral et al., 2012; Bardagi \& Albanaes, 2015). No entanto, quando tais intervenções acontecem (no âmbito de serviços-escola, projetos de extensão ou ações da gestão universitária, a partir de distintas modalidades de atendimento e diferentes referenciais teóricos) são ferramentas importantes para o desenvolvimento de habilidades profissionais e interpessoais, além de fomentar a orientação de carreira e a promoção de saúde, contribuindo para a redução da evasão (Bardagi \& Hutz, 2005; Morais, Mascarenhas, \& Ribeiro, 2010). Dessa maneira, intervenções baseadas em evidências tornam-se ferramentas necessárias para melhor adaptação ao ambiente acadêmico, para aumentar a eficácia da formação profissional e para promover a saúde desta população (Albanaes, Soares, \& Bardagi, 2015; Ambiel, Campos, \& Campos, 2017; Silva \& Bardagi, 2016).

O presente artigo tem por objetivo apresentar um conjunto de intervenções realizadas em uma universidade pública brasileira, enfocando dimensões relacionadas ao planejamento de carreira, desenvolvimento de hábitos de estudo, e promoção da saúde mental dos universitários. A justificativa para o fomento de intervenções com esta população se dá, entre outros motivos, pela importância deste período da vida do universitário, marcado por diferentes desafios, expectativas e decisões (Ambiel et.al, 2016; Borges e De Andrade, 2014).

\section{Método}

As propostas de intervenção apresentadas são oriundas de três Projetos de Extensão com diferentes abordagens teóricas, mas com a mesma intenção de fomentar serviços de apoio à população de universitários: "Triagem Psicológica (Triapsi)", "Serviço de Atenção Psicológica ao Graduando (Sapsig)" e "Educação para a Carreira (E-car)", e no âmbito de um Projeto Institucional de Apoio Acadêmico: "Sou Universitário, e agora? Uma preparação psicológica para a vida acadêmica (Projeto SUA)". As atividades são desenvolvidas e supervisionadas por três docentes do curso de Psicologia da Universidade Federal do Espírito Santo. As ações acontecem no Núcleo de Psicologia Aplicada (serviço-escola) e em salas de aula de cursos de graduação da universidade, desde 2015, e contam com a participação de estudantes de graduação em Psicologia, devidamente capacitados e orientados para o desenvolvimento das atividades.

As propostas aqui descritas, além de capacitar estudantes de Psicologia para realizar intervenções psicológicas individuais e em grupo com população universitária, têm como objetivos principais: 1 . oferecer um espaço de compartilhamento e comunicação sobre questões da vida acadêmica ao jovem ingressante na universidade; 2 . possibilitar ao estudante o desenvolvimento de estratégias de enfrentamento às dificuldades inerentes à vida acadêmica; 3. contribuir para o desenvolvimento de habilidades e competências que permitam ao estudante atender às exigências da vida acadêmica e da carreira, em especial 
quanto às atividades de estudo; 4. construir um plano de carreira articulado com a avaliação das oportunidades no mercado de trabalho e das habilidades pessoais; e 5. contribuir com a redução de índices de evasão e retenção associados a questões psicossociais.

As intervenções dos projetos foram elaboradas com base nas premissas do Programa Nacional de Assistência Estudantil (Brasil, 2010), que tem como finalidade ampliar as condições de permanência dos estudantes na educação superior pública federal; e do Programa de Assistência Estudantil, vigente no âmbito da universidade, que visa incentivar ações de cunho psicossocial e socioeducativo visando à integração dos estudantes à vida universitária. Assim, as ações são desenvolvidas em parceria com a Pró-reitoria de Assuntos Estudantis e de Cidadania (Proaeci) da universidade, que inicialmente demandou a parceria com o Curso de Psicologia, tendo em vista que sua equipe técnica de assistentes sociais e psicólogos não conseguia atender ao quantitativo das demandas dos estudantes. Periodicamente, são feitas reuniões entre as equipes dos projetos e a equipe técnica da Proaeci, a fim de detectar demandas de atendimento que chegam a esse setor da universidade e alinhar as propostas dos projetos a essas demandas.

Nesse contexto, entende-se que cuidar da saúde mental do universitário e do desenvolvimento de sua carreira é também ampliar as condições de sua permanência na universidade, tendo em vista a interface entre os processos de saúde-doença e o desempenho acadêmico e profissional. Os projetos aqui descritos beneficiaram, em seu conjunto, nos anos de 2015 a 2017, a 705 universitários. A seguir apresentam-se as estruturas das propostas, contemplando a formação dos discentes para a atuação nos projetos e as atividades desenvolvidas em cada uma das propostas de intervenção.

\section{Formação de discentes e articulação entre ensino, pesquisa e extensão}

Destaca-se que o processo de oferta de serviços de atenção aos universitários ocorre dentro de uma universidade pública, sendo a mesma comprometida com a articulação entre ensino, pesquisa e extensão. Assim, a participação dos alunos de graduação em Psicologia na condução das atividades dos projetos de extensão exige formação e treinamento específico, e está alinhada com o desenvolvimento das competências previstas nas Diretrizes Curriculares Nacionais para Cursos de Graduação em Psicologia (Ministério da Educação, 2011). Em todos os projetos os estudantes de graduação em Psicologia realizam um processo de seleção para ingresso, com critérios próprios, tendo em vista a adequação das atividades desenvolvidas ao período em que o estudante se encontra e as disciplinas já cursadas. Em seguida, os graduandos passam por um período de capacitação antes do início das atividades.

Em cada projeto, a capacitação se dá de uma maneira diferente. No âmbito dos projetos Triapsi e Sapsig, devido a sua interface, são ofertados conjuntamente cursos de extensão para os ingressantes, com vistas a contemplar aspectos teóricos e práticos das atividades a serem desenvolvidas nesses dois projetos. No ano de 2015 foi ofertado o curso: "Capacitação em Acolhimento, Triagem e Técnicas Clínicas", em 2016 o curso: "Fundamentos da Atuação Clínica: Abordagens Interpessoais e Dinâmicas no Atendimento ao Universitário" e em 2017: "Instrumento de Avaliação e Técnicas de Intervenção com Universitários" cada um deles com 20 horas de duração, todos registrados e certificados pela Pró-reitoria de Extensão da universidade.

No projeto SUA, a capacitação se deu por meio da discussão coordenada de textos teóricos e metodológicos no campo da Psicologia Grupal e de treinamentos vivenciais para aplicação de técnicas de condução de grupos psicodramáticos. Já no projeto Educação para a Carreira (E-car), os graduandos receberam, por um período de dois meses (8 encontros), uma formação teórica e técnica sobre como realizar o trabalho de planejamento de carreira e desenvolvimento de estratégias de enfrentamento da ansiedade frente a esse contexto.

No que concerne à dimensão da pesquisa, os projetos são comumente estruturados com base em conhecimentos científicos produzidos por pesquisadores nacionais e internacionais, tendo a participação de bolsistas de iniciação científica e por vezes alunos de pós-graduação (mestrado e doutorado). No Projeto Sapsig foram desenvolvidas duas pesquisas atreladas ao Projeto de Extensão "Estresse, ansiedade e estratégias de enfrentamento de provas escolares: um estudo com universitários" (no prelo).e "Avaliação do resultado da participação de universitários em um grupo de controle de ansiedade e enfrentamento do estresse" (Ramos, Kuster, Lucas \& Nascimento, 2019). Já o projeto E-Car se utiliza de instrumentos psicométricos adaptados pelo grupo de pesquisa "Laboratório de Avaliação e Mensuração Psicológica (Labamp), como por exemplo, a Escala de Afetos de Carreira (De Andrade, Pissaia, Silva, \& Oliveira, 2016).

Entre 2015 e 2017 foram capacitados 86 estudantes de graduação em Psicologia, que participaram das atividades dos projetos supervisionados semanalmente pelo docente responsável. Além da importância da formação de recursos humanos em Psicologia, destaca-se a importância 
do apoio da universidade às atividades desenvolvidas por meio da concessão de bolsas em todos os projetos, para alguns dos estudantes de Psicologia participantes. A seguir são descritos os objetivos e a metodologia de cada um dos projetos.

\section{Projeto "Sou Universitário e agora? Uma preparação psicológica para a vida acadêmica" (SUA)}

O Projeto SUA consistia na oferta de oficinas para estudantes ingressantes de diversos cursos de graduação da universidade ( $1^{\circ}$ e $2^{\circ}$ períodos), a fim de oferecer aos universitários um espaço de discussão e troca de experiências quanto ao início da vida acadêmica, por meio de uma estratégia de intervenção interativa, breve e focada, com enfoque preventivo. O Projeto SUA aconteceu em 2 edições, uma no ano de 2015 (com duração de 6 meses) e outra no ano de 2016 (com duração de 10 meses), pois se tratava de proposta submetida à Edital específico de concorrência de projetos na universidade, no âmbito do Programa Institucional de Apoio Acadêmico, da Próreitoria de Graduação.

$\mathrm{Na} 1^{\text {a }}$ edição, de 2015, inicialmente, era feito o contato com os coordenadores dos diversos cursos de graduação e, após apresentação da proposta, eram agendados os encontros com os cursos interessados. As oficinas foram realizadas com a turma toda de um determinado curso e período, na própria sala de aula do curso, e em horário regular de aula, cedido por algum professor do curso, facilitando assim a adesão dos estudantes. O projeto alcançou 5 cursos de graduação (Gemologia, Letras Português, Letras Inglês, Psicologia, Serviço Social) e beneficiou 86 universitários; e as atividades foram conduzidas por 10 graduandos em Psicologia (bolsistas), em duplas, por oficina. $\mathrm{Na} 1^{\mathrm{a}}$ edição, as oficinas em cada curso contaram com dois encontros de duas horas cada, com as temáticas de "Psicodrama" e "Aprender a aprender"; já no ano de 2016, foi acrescentado mais um encontro com a temática de "Construção de uma Carreira", também com duas horas de duração.

$\mathrm{Na} 2^{\mathrm{a}}$ edição, de 2016 , a proposta foi um pouco modificada e a divulgação da Oficina de preparação para a vida acadêmica foi feita pelo mailing da Proaeci e estudantes de diversos cursos se inscreviam nos dias e horários oferecidos que atendiam a sua disponibilidade de tempo. Essa edição beneficiou 97 universitários em 6 oficinas realizadas, cada uma com três encontros, sendo conduzidas por sete graduandos bolsistas em Psicologia, também em duplas por oficina. Nessa edição, também foram acrescentadas entrevistas de follow-up com alguns dos participantes $(N=7)$, realizadas segundo o procedimento de amostragem por conveniência. A entrevista teve como objetivo mapear os efeitos da participação da oficina na vida dos estudantes.

As atividades desenvolvidas em cada um dos dias da Oficina de preparação para a vida acadêmica (ano 2015: $1^{\circ}$ e $2^{\circ}$ dias e ano 2016: $1^{\circ}, 2^{\circ}$ e $3^{\circ}$ dias) são descritas a seguir:

$1^{\circ}$ dia: Psicodrama. O psicodrama é uma abordagem da psicologia clínica que se caracteriza pelo uso de técnicas adaptadas do teatro com o objetivo de oferecer a indivíduos e grupos a oportunidade de vivenciar dinamicamente seus conflitos e por meio dessa vivência, reelaborar sentimentos e atitudes psicológicas (Monteiro, 1994). Neste dia de oficina, por meio das técnicas psicodramáticas, os estudantes eram levados a refletir sobre o que é ser universitário e as principais dificuldades e oportunidades nesse contexto, por meio da representação de situações como o "trote" e dos papeis de aluno e professor.

$2^{\circ}$ dia: "Aprender a aprender". Este encontro tinha por objetivo desenvolver ferramentas necessárias para a criação de hábitos de estudo, por meio de atividades e discussões focadas em: discutir o significado de estudar e as diferenças entre o estudo no ensino médio e no superior; desenvolver estratégias mais eficientes de estudo; identificar aspectos facilitadores na organização das atividades de estudo; identificar dificuldades pessoais que estejam interferindo negativamente nos hábitos de estudo; conhecer e adotar princípios de gerenciamento do tempo (Basso, Graf, Lima, Schmidt, \& Bardagi, 2013; Rodrigues, 2005).

$3^{\circ}$ dia: "Construção de uma Carreira". Este encontro tinha por objetivo apresentar aos universitários as novas perspectivas de carreira e desafios para empregabilidade. O conteúdo teórico do encontro era fundamentado nas perspectivas de carreira desenvolvimentistas para o século XXI (Savickas et al., 2009), sendo a oficina organizada num primeiro momento para explicitação do modelo tradicional de carreira versus os novos modelos, seguida por uma discussão ampliada sobre competências e estratégias para formação de um diferencial competitivo. $\mathrm{Na}$ sequência da oficina, era destinado um tempo final para que os participantes expusessem suas dúvidas sobre futuro profissional, aspectos de ansiedade e possíveis soluções para estes contextos.

\section{Projeto de Acolhimento e Triagem Psicológica (Triapsi)}

Por meio do Projeto de Extensão Triapsi, é possibilitado, ao universitário interessado nas propostas de intervenção oferecidas no âmbito do Sapsig, realizar uma avaliação de suas demandas e, em seguida, é feito o direcionamento da modalidade de atendimento que mais se 
ajusta às suas necessidades e perfil. A acolhida e a triagem são realizadas de forma individual, em um processo com 2 a 5 encontros de até 50 minutos cada, a partir da perspectiva da Abordagem Centrada na Pessoa (Rogers, 2001), e é pré-requisito para a participação nas atividades oferecidas pelo Projeto Sapsig. De 2015 a 2017, 39 graduandos em Psicologia conduziram as entrevistas de acolhimento.

A acolhida às demandas dos universitários por meio de entrevista inicial de triagem tem um papel importante, pois permite identificar as queixas do estudante, suas demandas, e qual modalidade de atendimento melhor se adequa às particularidades de cada caso (Cavalheiro et al., 2012). Outro benefício da entrevista de triagem é a possibilidade de um encaminhamento assertivo, que permite que os universitários se engajem no atendimento, diminuindo assim os índices de evasão dos programas de intervenção.

A fim de auxiliar a realização da entrevista de triagem e a tomada de decisão quanto ao encaminhamento das demandas foi desenvolvido, no âmbito deste projeto de extensão, o Instrumento de Triagem Psicológica (ITP). O ITP baseia-se na abordagem da Psicoterapia Integrativa (Stricker \& Gold, 2013) e avalia cinco dimensões para a direção do tratamento, a saber: severidade, motivação, complexidade, recursos e estilos de coping. Essas cinco dimensões são assim definidas: 1 . Severidade: refere-se à extensão dos efeitos da queixa principal na vida do universitário; 2. Motivação: refere-se ao grau em que o indivíduo se encontra motivado a envolver-se com o tratamento; 3. Complexidade: aponta para o quanto o problema já se tornou parte do modo como o indivíduo lida com o seu cotidiano; 4. Recursos: denotam a capacidade de reflexão do indivíduo, o foco na compreensão do seu problema e a percepção da relação dos problemas com seus hábitos, valores e comportamentos; e, 5. Estilos de coping: apontam para a forma habitual do universitário enfrentar os desafios cotidianos de sua vida (Beutler \& Clarkin, 2014).

O ITP não tem o objetivo de compor perfis estatísticos ou uma tipologia particular generalizável para uma população mais ampla, fora do contexto clínico. Ao contrário, constitui-se em um instrumento com o propósito de tomada de decisões circunscritas ao contexto clínico, visando um encaminhamento dos casos mais fundamentado e sistemático. Com a experiência de aplicação do instrumento, percebeu-se que estudantes que apresentavam alta complexidade e alta severidade se beneficiavam pouco das propostas breves de intervenção em grupo (as oficinas descritas adiante), sendo mais recomendado seu encaminhamento para a psicoterapia individual. Assim, o ITP tornou-se uma ferramenta importante para avaliação do universitário e para a tomada de decisão para qual proposta de intervenção encaminhá-lo.

\section{Serviço de Atenção Psicológica ao Graduando (Sapsig)}

As atividades desenvolvidas no âmbito do Sapsig ocorrem por meio de oficinas e psicoterapia individual, conduzidas a partir do referencial da clínica analítico-comportamental (Borges \& Cassas, 2012). A divulgação das propostas é feita via mailing da Proaeci, e, após passar pelo acolhimento e triagem no Triapsi, o universitário é encaminhado para a proposta que mais atende a suas necessidades e demandas. Nos anos de 2015 a 2017 participaram das 17 oficinas realizadas 279 universitários, que foram conduzidas por 23 graduandos em Psicologia (a partir do $6^{\circ}$ período), em duplas de terapeutas. Todas as oficinas contam com 8 encontros de 1 hora e meia cada, com, no máximo, 15 participantes. Os objetivos de cada uma das oficinas são descritos a seguir.

\section{Oficina de Habilidades Sociais}

A Oficina de Habilidades Sociais tem por objetivo desenvolver, por meio de técnicas vivenciais, o repertório de habilidades interpessoais dos participantes, com a intenção de melhorar sua competência nas situações sociais que exijam: iniciar e manter conversações; falar em público; defender os próprios direitos; expressar opiniões pessoais, pensamentos e/ou sentimentos, sejam eles positivos (alegria, amor, elogio) ou negativos (raiva, ressentimento, crítica); dentre outros (Del Prette \& Del Prette, 2013). Tais habilidades são importantes para a vida pessoal do universitário na medida em contribuem para a redução da ansiedade e que seu ensino pode facilitar padrões de comportamentos pró-sociais que tendem a reduzir efetivamente a ocorrência de problemas de comportamento (Garcia, Bolsoni-Silva, \& Nobile, 2015).

Do ponto de vista acadêmico, habilidades sociais bem desenvolvidas estão relacionadas ao alcance de metas de aprendizagem (Feitosa, Del Prette, \& Del Prette, 2012) e a um bom desempenho acadêmico e menores índices de evasão (Ferreira et al., 2014). A literatura apresenta diversas propostas dessa natureza desenvolvidas com universitários e com bons resultados (Pureza, Rusch, Wagner, \& Oliveira, 2012; Lopes, Dascanio, Ferreira, Del Prette, \& Del Prette, 2017).

\section{Oficina de Orientação aos Estudos}

O processo de aprendizagem é complexo e envolve variáveis do professor, do aluno e da instituição de ensino; no caso do aluno do ensino superior, é preciso ter autonomia para buscar informações quando necessário e atuar ativamente na construção do conhecimento. 
Assim, uma das exigências identificadas no contexto acadêmico é a necessidade de obter habilidades de autorregulação, que consiste na necessidade do estudante estabelecer objetivos de aprendizagem, ter conhecimento de quais são suas capacidades e dificuldades em relação a esse processo e implementar estratégias adequadas de aprendizado (Sampaio, Polydoro, \& Rosário, 2012).

A Oficina de Orientação aos Estudos tem por objetivo trabalhar com os universitários as ferramentas necessárias para a criação de hábitos de estudo, por meio de atividades, técnicas de grupo e discussões focadas em: desenvolver estratégias mais eficientes de estudo; aprender a gerir o tempo, identificar aspectos facilitadores na organização das atividades de estudo; identificar dificuldades pessoais que estejam interferindo negativamente nos hábitos de estudo e combater a procrastinação acadêmica (Sampaio \& Bariani, 2011).

Oficina de Controle da Ansiedade e Enfrentamento do Estresse

Estados emocionais intensos podem interferir na atenção, concentração e na capacidade de aprendizagem; assim, para alguns estudantes, os principais obstáculos para um bom desempenho acadêmico não são dificuldades de natureza cognitiva, mas de ordem emocional, que podem ser contornadas à medida que o estudante aprenda a lidar melhor com elas, por meio da adoção de estratégias de enfrentamento eficazes (Oliveira, Carlotto, Vasconcelos, \& Dias, 2014). Assim, a Oficina de Controle de Ansiedade e Enfrentamento do Estresse visa a auxiliar estudantes cujo desempenho acadêmico seja prejudicado pelo excesso de ansiedade e de estresse vivenciados cotidianamente ou nas situações de provas e avaliações escolares (Bardagi \& Hutz, 2011).

\section{Oficinais com temáticas específicas}

Estas oficinas são desenvolvidas a partir de demandas específicas de alguns cursos de graduação da universidade, que entram em contato com o Sapsig, buscando uma intervenção para resolução de diversos problemas como: conflitos interpessoais e de relacionamento em turmas, problemas de preconceito e exclusão social, dentre outros. As atividades e metodologia das oficinas são delineados a partir da avaliação inicial da demanda.

\section{Psicoterapia individual}

Além das dificuldades estritamente ligadas às competências acadêmicas e de estudo, sabe-se que inúmeras dificuldades de ordem emocional (conflitos pessoais e familiares, dentre outros) podem atrapalhar o bom desempenho do aluno em seu curso superior. $\mathrm{O}$ atendimento psicológico individual visa a auxiliar o estudante na resolução de diversas demandas pessoais (conflitos familiares, problemas na faculdade) e profissionais (dúvidas em relação à escolha do curso, por exemplo) de acordo com a necessidade de cada caso. Os atendimentos são realizados semanalmente, com duração média de 50 minutos cada, e pelo tempo necessário à resolução da questão trazida pelo estudante. Nos anos de 2015 a 2017 foram beneficiados 43 estudantes em psicoterapia (com duração de um mês a dois anos, conforme a necessidade do caso).

\section{Projeto "Educação para a Carreira (E-car)"}

Além de ajudar o universitário em sua adaptação à vida acadêmica, no desenvolvimento de habilidades necessárias ao estudo e ao sucesso profissional, na resolução de conflitos interpessoais, é necessário prepará-lo para a realidade que irá encontrar quando concluir seu curso superior e ingressar no mercado de trabalho. A Educação para a Carreira consiste em uma modalidade de orientação profissional, desenvolvida de forma sistemática, e procura estabelecer para os universitários a ligação entre o que se aprende na escola/universidade e o mundo do trabalho (Munhoz \& Melo-Silva, 2011).

No Projeto de Extensão "Educação para a Carreira (E-CAR)" são realizadas intervenções diferenciadas no nível individual e grupal (curso, palestras, treinamento, seminários) que atendam às necessidades da população-alvo (estudantes de graduação nos últimos períodos do curso), a fim de contribuir para a construção dos projetos profissionais e de vida dos participantes. As atividades são desenvolvidas com base no modelo desenvolvimentista de carreira de Savickas (Savickas et al., 2009) e de carreira proteana (Hall, 2004).

Atendendo a uma perspectiva construtivista e individual de carreira, as intervenções são baseadas em processos de autoconhecimento, incremento de habilidades de gerenciamento profissional, desenvolvimento de empregabilidade e planejamento de vida-carreira. As intervenções de desenvolvimento de carreira, tanto no formato individual quanto em grupo são organizadas no formato de encontros fechados, de quatro a seis sessões, uma por semana. Nesses encontros são realizadas as seguintes atividades:

Palestra para apresentação do projeto. Um evento de abertura realizado pela equipe e o supervisor responsável é realizada em cada início de semestre, com o objetivo de sensibilizar os alunos-clientes, bem como apresentar 
a sistemática do serviço de carreira. O tema da palestra comumente gira em termos de problemáticas de carreira, como por exemplo, empregabilidade em momento de crise econômica, construção de uma carreira de sucesso, decisões de carreira entre outros. Tais eventos são importantes para sensibilização dos universitários sobre a importância de investir recursos no processo de planejamento e desenvolvimento de carreira. A segunda parte, relacionada à apresentação da sistemática do trabalho tem um papel importante na organização e operacionalização do serviço. Nesta etapa são discutidos aspectos como faltas, horários, exercícios e duração do trabalho.

Avaliação Integrativa de Aspectos Psicossociais Ligados à Carreira. Procedimento no qual se busca conhecer diferentes características do cliente, como por exemplo: interesses pessoais e profissionais, valores sobre trabalho, influência da família no processo de carreira, conhecimento do mercado de trabalho, nível de estresse e ansiedade com aspectos de carreira, dentre outros. Neste encontro há possibilidade de uso de instrumentos psicológicos padronizados, sendo utilizados em algumas oportunidades testes psicológicos para avaliação de personalidade, autoeficácia e saúde (depressão, ansiedade e estresse).

Encontros de intervenção (individual ou em grupo). Comumente organizados em três encontros nos quais são trabalhados os seguintes tópicos; 1. Conhecendo o mercado de trabalho e as ferramentas on-line para formação profissional; 2. Desenvolvendo estratégias de empregabilidade para cenários de incerteza; 3 . Portfólio de carreira; e 4. Dimensões da vida no trabalho e não-trabalho.

Conclusão dos grupos e avaliação do impacto do projeto. Ao final de cada grupo é realizado um encontro final para compartilhamento das experiências de desenvolvimento de carreira. Comumente nos encontros são aplicados questionários qualitativos centrados nos aspectos de percepções de mudanças durante as intervenções realizadas.
As atividades dos Projetos de Extensão Triapsi, Sapisg e E-car são permanentes e continuam em funcionamento na universidade. A seguir apresentamos um breve panorama da avaliação das intervenções realizadas e são discutidas as implicações dos projetos no atendimento às demandas psicológicas dos universitários e para a formação de recursos humanos em Psicologia.

\section{Resultados}

Nas oficinas desenvolvidas no âmbito do Sapsig, a avaliação das intervenções é realizada de maneira coletiva ou individual, a partir de dinâmicas de grupo, ou ainda a partir de questionários semiabertos, com perguntas que avaliam o grau de adesão do participante na oficina, motivação para participar do serviço, mudanças percebidas ao longo da realização dos encontros e sugestões de mudança. Destaca-se que o processo de implementação das avaliações não ocorreu desde o primeiro ano do projeto, nem tão pouco em todas as atividades realizadas; todavia, é possível apresentar algumas impressões gerais da avaliação das intervenções.

A avaliação das oficinas do Sapsig foi realizada por 83 participantes, contemplando os anos de 2015 a 2017. Os universitários avaliaram as intervenções a partir de um questionário semiaberto, contendo 4 perguntas fechadas sobre percepções gerais da oficina (ex. qual seu grau de aproveitamentos da oficina realizada? como avalia suas habilidades de controlar o estresse e a ansiedade após a participação na oficina?) e 3 perguntas abertas para encaminhamento de sugestões e avaliação da equipe (ex. como avalia a atuação das alunas-terapeutas que conduziram a oficina?). A apuração dos escores médios das avaliações das perguntas fechadas foi feita por uma escala de 5 pontos, com chaves de resposta para mensuração da intensidade das mudanças e afetos vivenciados $(1$ - pouco a 5 - muito). A Tabela 1 apresenta os resultados descritivos do questionário fechado.

Tabela 1

Avaliação das oficinas do Sapsig $(N=83)$

\begin{tabular}{lcc}
\hline \multicolumn{1}{c}{ Item } & Média & $\begin{array}{c}\text { Desvio- } \\
\text { padrão }\end{array}$ \\
\hline 1. Quando participei dos encontros da oficina, acho que aprendi sobre os temas discutidos... & 4,39 & 0,66 \\
2. Em relação a minha participação na oficina, considero que me envolvi... & 4,18 & 0,60 \\
3. Em relação aos sintomas que tinha antes da participação na oficina, sinto que mudei... & 4 & 0,58 \\
4. Me senti confortável em participar dos encontros... & 4,89 & 0,34 \\
\hline
\end{tabular}

Devido ao não uso de instrumentos padronizados, os resultados tornam a análise pouco precisa e geral; no entanto, a partir de 2018 estão sendo adotados instrumentos padronizados aplicados antes e após a participação dos 
universitários na oficina, a fim de verificar as diferenças entre esses dois momentos. Todavia, considerando os escores variando de 1 a 5 , e o fato dos 4 valores de médias apresentarem valor acima do ponto médio $(2,5)$, constata-se que a oficinas foram percebidas de maneira positiva no que se refere aos aspectos avaliados. Os dados das questões abertas do mesmo questionário corroboram essa análise ao sinalizar o bom desempenho dos alunos terapeutas, a importância dos temas discutidos e o adequado suporte recebido ao longo dos atendimentos.
Em relação ao Projeto SUA, a avaliação foi realizada a partir de um formulário construído pelos integrantes do projeto. $\mathrm{O}$ formulário continha três eixos centrais (qualidade da oficina, envolvimento na oficina e avaliação da equipe), avaliados em dimensões específicas (ex. pontualidade, originalidade, envolvimento, dentre outros). Os itens foram respondidos em escala que variava de 1 (muito ruim) a 7 (excelente). A média o desvio padrão das respostas de 113 participantes das oficinas do SUA é apresentada na Tabela 2.

Tabela 2

Avaliação das oficinas do Projeto SUA $(N=113)$

\begin{tabular}{lcc}
\hline \multicolumn{1}{c}{ Item } & Média & $\begin{array}{c}\text { Desvio- } \\
\text { padrão }\end{array}$ \\
\hline Eixo 1. Que nota você dá para a oficina nos seguintes critérios: & 6,51 & 0,36 \\
Pontualidade & 6,71 & 0,18 \\
Dinamismo & 6,38 & 0,36 \\
Profundidade & 6,59 & 0,27 \\
Originalidade & 6,53 & 0,35 \\
Foi útil & 6,71 & 0,34 \\
Foi interessante & 6,68 & 0,26 \\
Foi dinâmica & 6,21 & 0,45 \\
Foi intensa & & \\
& & 0,49 \\
Eixo 2. Que nota você dá para sua participação na oficina nos seguintes critérios: & 0,26 \\
Assiduidade & 6,28 & 0,37 \\
Pontualidade & 6,15 & 0,25 \\
Interesse & 6,59 & 6,65 \\
Envolvimento & & \\
Eixo 3. Que nota você dá para os facilitadores da oficina nos seguintes critérios: & & 0,26 \\
Domínio do grupo & 6,68 \\
Dinamismo & 6,66 & 0,25 \\
Envolvimento emocional com o grupo & 6,35 & 0,39 \\
Assiduidade e pontualidade & 6,67 & 0,25 \\
\hline
\end{tabular}

Verifica-se que as médias de todos os itens avaliados estão acima de 6 , indicando, assim, uma avaliação muito positiva destas oficinas. Nas entrevistas de seguimento do Projeto SUA, realizadas em média 2 meses após o término das oficinas, foram destacados como principais aspectos positivos do projeto, dentre outros: acolhida aos calouros enquanto novos integrantes da universidade, oferecimento de um espaço para discussão de temas e problemas que não eram abordados em outros contextos, possibilidade de aplicação do aprendizado no que se refere a estudar para as provas. Como aspectos negativos foram apontados: o pouco tempo das oficinas e a necessidade de continuidade desse acompanhamento com os universitários. Todos os participantes da entrevista relataram que a oficina os auxiliou a lidar com as dificuldades da vida acadêmica e social, e afirmaram a necessidade de mais projetos que abordem as questões relacionadas à vida acadêmica, carreira, relacionamento interpessoal e bem-estar do universitário.

Em relação aos graduandos em Psicologia que atuaram nos projetos, os formulários que compõem os instrumentos da própria universidade para avaliação dessas atividades revelaram uma percepção positiva da contribuição da experiência, como se vê nos seguintes relatos:

"Foi gratificante participar deste Projeto Extensão, tanto profissionalmente quanto pessoalmente. Tive oportunidade de aprimorar habilidades para atuação 
em atendimento psicoterapêutico individual, condução de grupos e trabalho em equipe, integrando conhecimentos teóricos e práticos. Excelente Projeto de Extensão, tanto para os alunos integrantes quanto em relação ao papel social que ele mantém na universidade, que é o de atendimento gratuito aos graduandos." (extensionista do Sapsig).

"Executar essas oficinas foi de extrema importância para o meu desenvolvimento acadêmico, visto que estava cursando o nono período, no decorrer do projeto, e ainda não havia tido a oportunidade de executar oficinas diretamente ligadas a esses temas. Estar em outros cursos, escutando outras realidades podendo problematizá-las e ajudar minimamente também foram de total relevância." (graduanda do SUA).

\section{Discussão}

A política nacional de assistência estudantil (Brasil, 2010) preconiza ações desenvolvidas em nível institucional que favoreçam a permanência do estudante no ensino superior sendo a atenção à saúde e a melhoria do desempenho acadêmico dois de seus objetivos. Os diferentes projetos aqui descritos buscam colaborar no cumprimento dos objetivos de tal política, mediante a oferta de atenção psicológica ao universitário em diferentes serviços de apoio, integrados entre si, e que articulam ensino, pesquisa e extensão.

Essas propostas de intervenção com universitários visam, em seu conjunto, contribuir para a adaptação do estudante à vida acadêmica, no desenvolvimento da autonomia no seu processo de aprendizagem, e na gestão da própria carreira (Munhoz \& Melo-Silva, 2011; Oliveira et al., 2014; Sampaio et al., 2012). Tais intervenções podem auxiliar no enfrentamento dos inúmeros desafios vivenciados na trajetória do ensino superior, e auxiliar na minimização do adoecimento psicológico e na promoção da saúde mental do estudante, conforme outros trabalhos da área (Amaral et al., 2012; Ferreira et al., 2014; Lantyer et al., 2016).

Além disso, o volume das atividades realizadas e a variedade de metodologias e referenciais teóricos utilizados fornecem uma amostra da importância desse tipo de intervenção psicológica para a população de 706 universitários beneficiados. Pela perspectiva dos usuários do serviço, é uma forma da universidade, na qual os projetos são realizados, oferecer suporte institucional aos diferentes desafios que enfrentam no seu percurso acadêmico (Moreno \& Soares, 2014). Tais ações podem contribuir para a diminuição da retenção e evasão no ensino superior devido a fatores psicossociais (Ambiel et al., 2016; Bardagi \& Hutz, 2005).
No entanto, apesar do número de estudantes beneficiados, as ações ainda são quantitativamente insuficientes para abarcar o conjunto das necessidades da população dessa universidade, composta por cerca de 20.000 estudantes de graduação, conforme indica a equipe técnica da Proaeci e se visualiza na lista de espera para participação nas oficinas e atendimentos individuais dos projetos aqui descritos. Assim, novos projetos devem ser propostos de forma a atender a totalidade da comunidade acadêmica que procura por serviços de atenção psicológica.

Neste trabalho destaca-se, também, a importância da formação e preparação dos 86 estudantes de graduação em Psicologia, envolvidos na condução das atividades, contribuindo para o desenvolvimento das competências previstas nas diretrizes curriculares para Cursos de Graduação em Psicologia (Ministério da Educação, 2011). Ao lidarem com diferentes nuances da problemática da adaptação à vida universitária, os projetos permitem a tais graduandos a exploração de possibilidades de atuação próximas ao mercado de trabalho (como no caso daqueles que optarem pelas carreiras técnicas nas universidades públicas ou privadas que lidarão diretamente com o público universitário), além de fortalecerem a construção de uma identidade profissional.

Uma das limitações das propostas apresentadas neste artigo é a falta de uma avaliação sistemática sobre a eficácia das intervenções desenvolvidas. Este problema foi observado ao longo dos três anos de existência dos projetos, e tem sido aprimorado desde então. Com base na experiência prática, sugere-se para pesquisadores que possam se interessar por intervenções com universitários a realização de avaliação de pré e pós intervenção, permitindo a comparação de indicadores de mudança; e a adoção de estratégias mistas de avaliação, envolvendo entrevista individual, coletiva ou técnicas de grupos focais que acrescentem um enfoque qualitativo aos dados de medidas mais objetivas. Algumas dessas propostas já estão sendo adotadas para as intervenções iniciadas em 2018. Além disso, a inclusão de escalas psicológicas e inventários comportamentais com evidências de validade e precisão para o contexto brasileiro (Audibert \& Teixeira, 2015; Borges, De Andrade, Oliveira, \& Guerra, 2015; De Andrade, Pissaia, Silva, \& Oliveira, 2016; Silva \& De Andrade, 2016; Oliveira et al., 2010; Soares, Mourão, Valory, \& Mello, 2011), de acordo com os objetivos das intervenções, também pode ser útil.

\section{Considerações finais}

Para futuros professores, psicólogos e profissionais da área de gestão educacional que tenham interesse em realizar 
atividades de natureza interventiva e de suporte psicológico com universitários, é importante destacar o papel central da administração universitária na articulação das políticas de assistência estudantil por meio da parceria dos projetos desenvolvidos com os setores da universidade que implementam tais políticas. Essa articulação deve ocorrer tanto na dimensão de fomento, envolvendo bolsas para alunos dos projetos, quanto na disponibilização de material e espaço físico para realização das atividades, mas principalmente na sensibilização da comunidade acadêmica para importância dos temas tratados nestas intervenções.
Assim, os órgãos de gestão educacional possuem competência para o alinhamento de estratégias e recursos que viabilizem não só o sucesso das ações psicossociais dirigidas aos graduandos, mas também a manutenção do compromisso ético das instituições públicas de ensino superior com o futuro do país. Espera-se que as propostas aqui descritas contribuam com o avanço da produção de conhecimento na área de atendimento psicológico ao universitário e favoreçam a implantação de outros serviços que visem atender às demandas e necessidades da população universitária no Brasil.

\section{Referências}

Albanaes, P., Soares, F. M. S., \& Bardagi, M. P. (2015). Programas de tutoría y mentoria em universidades brasilenas: um estudo bibliométrico. Revista de Psicologia, 33(1), 20-56. Recuperado em 20 de maio de 2018, de http://revistas. pucp.edu.pe/index.php/psicologia/article/view/11170

Amaral, A. E. V., Silva, M. A., Lopes, F. L, Leite, C. A., Luca, L., \& Rodrigues, T. C. (2012). Programa de acolhimento a universitários: Relato de experiência em clínica-escola. Encontro: Revista de Psicologia. 15(23), 27-35. Recuperado em 25 de abril de 2016, de http://www.pgsskroton.com.br/seer/index.php/renc/article/view/2465/2362

Ambiel, R. A. M., Campos, M. I., \& Campos, P. P. T. V. Z. (2017). Análise da Produção Científica Brasileira em Orientação Profissional: Um Convite a Novos Rumos. Psico-USF, 22(1), 133-145. doi: 10.1590/1413-82712017220112

Ambiel, R. A. M., Santos, A. A. A., \& Dalbosco, S. N. P. (2016). Motivos de evasão, vivências acadêmicas e adaptabilidade de carreira em universitários. Psico (Porto Alegre), 47(4), 288-297. doi: 10.15448/1980-8623.2016.4.23872

Audibert, A., \& Teixeira, M. A. P. (2015). Escala de adaptabilidade de carreira: Evidências de validade em universitários brasileiros. Revista Brasileira de Orientação Profissional, 16(1), 83-93. Recuperado em 17 de junho de 2018, de http://pepsic.bvsalud.org/scielo.php?script=sci_arttext\&pid=S1679-33902015000100009 \& lng=pt\&tlng=pt.

Bardagi, M. P., \& Albanaes, P. (2015). Avaliação de Intervenções Vocacionais no Brasil: Uma Revisão da Literatura. Revista Brasileira de Orientação Profissional, 16(2), 123-135. Recuperado em 17 de junho de 2018, de http://pepsic. bvsalud.org/scielo.php?script=sci_arttext\&pid=S1679-33902015000200004 \&lng=pt\&tlng=pt.

Bardagi, M. P., \& Hutz, C. S. (2005). Evasão universitária e serviços de apoio ao estudante: uma breve revisão da literatura brasileira. Psicologia Revista, 14(2), 279-301. Recuperado em 15 de maio de 2015, de https://revistas. pucsp.br/index.php/psicorevista/article/view/18107/13463

Bardagi, M. P., \& Hutz, C. S. (2011). Eventos estressores no contexto acadêmico: Uma breve revisão da literatura brasileira. Interação em Psicologia, 15(1), 111-119.

Basso, C., Graf, L. P., Lima, F. C., Schmidt, B., \& Bardagi, M. P. (2013). Organização de tempo e métodos de estudos: oficinas com estudantes universitários. Revista Brasileira de Orientação Profissional, 14(2), 277-288. Recuperado em 30 de julho de 2016, de http://pepsic.bvsalud.org/scielo.php?script=sci_arttext\&pid=S1679-33902013000200012\&lng=p t\&nrm=iso

Beutler, L. E., \& Clarkin, J. F. (2014). Systematic Treatment Selection: Toward targeted therapeutic interventions. Abingdon: Routledge.

Borges, L. L. F., \& De Andrade, A. L. (2014). Preditores da carreira proteana: Um estudo com universitários. Revista Brasileira de Orientação Profissional, 15(2), 153-163. Recuperado em 17 de junho de 2018, de http://pepsic.bvsalud. org/ scielo.php?script=sci arttext\&pid=S1679-33902014000200006\&lng=pt\&tlng=pt.

Borges, L. L. F., De Andrade, A. L., Oliveira, M. Z., \& Guerra, V. M. (2015). Expanding and adapting the Protean Career Management Scale for university studentes (PCMS-U). The Spanish Journal of Psychology, 18, E103, 1-13. doi: $10.1017 /$ sjp.2015.83

Borges, N. B., \& Cassas, F. A. (2012). Clínica analítico-comportamental: Aspectos teóricos e práticos. Porto Alegre: Artes Médicas.

Brasil. (2010). Programa Nacional de Assistência Estudantil. Decreto n ${ }^{\circ} 7.234$ de 19 de Julho de 2010. Recuperado em 26 de abril de 2014, de http://www.planalto.gov.br/ccivil_03/_ato2007-2010/2010/decreto/d7234.htm

Cavalheiro, N. C., Garcia, B. G., Iwata, H., Júnior, J. P., Rosa, H. R., Valente, M. L. L. C., \& Migliorini, W. J. M. (2012). Triagem interventiva: a caracterização de uma demanda. Revista SBPH, 15(2), 3-16. Recuperado em 23 de fevereiro de 2016, de http://pepsic.bvsalud.org/scielo.php?script=sci_arttext\&pid=S1516-08582012000200002 \&lng=pt\&tlng=pt 
De Andrade, A. L., Pissaia, A. T., Silva, M., Z., \& Oliveira, M. Z. (2016). Características proteanas e afetos sobre carreira de estudantes de Psicologia. Estudos de Psicologia (Campinas), 33(4), 677-688. doi: 10.1590/1982-02752016000400011

Del Prette, A., \& Del Prette, Z. A. P. (2013) Programas eficaces de entrenamiento en habilidades sociales basados en métodos vivenciales. Apuntes de Psicología, 31(3), 67-76.

Eckschmidt, F., Andrade, A. G., \& Oliveira, L. G. (2013). Comparação do uso de drogas entre universitários brasileiros, norte-americanos e jovens da população geral brasileira. Jornal Brasileiro de Psiquiatria, 62(3), 199-207. Recuperado em 20 de outubro de 2017, de http://www.scielo.br/pdf/jbpsiq/v62n3/04.pdf

Feitosa, F. B., Del Prette, Z. A. P., \& Del Prette, A. (2012). Social skills and academic achievement: The mediating function of cognitive competence. Temas em Psicologia, 20(1), 61-70. Recuperado em 7 de dezembro de 2016, de http://pepsic.bvsalud.org/scielo.php?script=sci_arttext\&pid=S1413-389X2012000100006 \&lng=pt\&tlng=

Ferreira, V. S., Oliveira, M. A., \& Vandenberghe, L. (2014). Efeitos a curto e a longo prazo de um grupo de desenvolvimento de habilidades sociais para universitários. Psicologia: Teoria e Pesquisa, 30(1), 73-81. doi: 10.1590/S0102-37722014000100009

Friedrich, A. C. D., Macedo, F., \& Reis, A. H. (2015). Vulnerabilidade ao stress em adultos jovens. Revista Psicologia Organizações e Trabalho, 15(1), 59-70. DOI: dx.doi.org/10.17652/rpot/2015.1.499

Garcia, V. A., Bolsoni-Silva, A. T., \& Nobile, G. F. G. (2015). A interação terapêutica em intervenções com universitários com Transtorno de Ansiedade Social. Psicologia: Ciência e Profissão, 35(4), 1089-1105. doi: 10.1590/1982-3703000842014.

Hall, D. T. (2004). The protean career: A quarter-century journey. Journal of Vocational Behavior, 65(1), 1-13. doi: 10.1016/j.jvb.2003.10.006

Lantyer, A. S., Varanda, C. C., Souza, F. G., Padovani, R. C., \& Viana, M. B. (2016). Ansiedade e qualidade de vida entre estudantes universitários ingressantes: avaliação e intervenção. Revista Brasileira de Terapia Comportamental e Cognitiva, 18(2), 4-19. Recuperado em 13 de junho de 2017, de http://www.usp.br/rbtcc/index.php/RBTCC/article/ view/880/476

Lopes, D. C., Dascanio, D., Ferreira, B. C., Del Prette, A., \& Del Prette, Z. A. P. (2017). Treinamento de habilidades sociais: Avaliação de um programa de desenvolvimento interpessoal profissional para universitários de ciências exatas. Interação em Psicologia, 21(1), 55-65. DOI: 10.5380/psi.v21i1.36210

Ministério da Educação. (2011). Resolução $n^{\circ} 5$ de 15 de março de 2011, Institui as Diretrizes Curriculares Nacionais para os cursos de graduação em Psicologia, estabelecendo normas para o projeto pedagógico complementar para a Formação de Professores de Psicologia. Recuperado em 17 de junho de 2012, de http://portal.mec.gov.br/index. php?option=com_docman\&view=download\&alias=7692-rces005-11-pdf\&Itemid=30192

Monteiro, R. F. (1994). Jogos dramáticos. São Paulo: Ágora.

Morais L. M., Mascarenhas, S. A. N., \& Ribeiro, J. L. P. (2010). Diagnóstico do estresse, ansiedade e depressão em universitários: desafios para um serviço de orientação e promoção da saúde psicológica na universidade, um estudo com estudantes da UFAM-Brasil. Amazônica, Revista de Psicopedagogia, Psicologia Escolar e Educação, 4(1), 55-76. Recuperado em 30 de junho de 2016, de https://dialnet.unirioja.es/servlet/articulo?codigo $=4028883$

Moreno, P. F., \& Soares, A. B. (2014). O que vai acontecer quando eu estiver na universidade? Expectativas de jovens estudantes brasileiros. Aletheia, 45, 114-127. Recuperado em 20 de janeiro de 2016 de, http:/pepsic.bvsalud.org/ scielo.php?script=sci_arttext\&pid=S14 13-03942014000200009\&lng=pt\&tlng=pt

Munhoz, I. M. S., \& Melo-Silva, L. L. (2011). Educação para a Carreira: Concepções, desenvolvimento e possibilidades no contexto brasileiro. Revista Brasileira de Orientação Profissional, 12(1), 37-48. Recuperado em 30 de abril de 2014, de http://pepsic.bvsalud.org/scielo.php?script=sci_arttext\&pid=S1679-33902011000100006

Oliveira, C. T., Carlotto, R. C., Vasconcelos, S. J. L., \& Dias, A. C. C. (2014). Adaptação acadêmica e coping em estudantes universitários brasileiros: uma revisão de literatura. Revista Brasileira de Orientação Profissional, 15(2), 177-186. Recuperado em 23 de abril de 2016, de http://pepsic.bvsalud.org/scielo. php?script=sci_arttext\&pid=S1679-33902014000200008\&lng=pt\&tlng=pt

Oliveira, M. Z., Zanon, C., Silva, I. S., Pinhatti, M. M., Gomes, W. B., \& Gauer, G. (2010) Validação da versão brasileira da Escala de Atitudes de Carreira Sem-Fronteiras. Arquivos Brasileiros de Psicologia, 62(3), 106-114. Recuperado em 10 de junho de 2018, de http://pepsic.bvsalud.org/scielo. php?script=sci_arttext\&pid=S1809-52672010000300012\&lng=pt.

Padovani, R. C., Neufeld, C. B., Maltoni, J., Barbosa, L. N. F., Souza, W. F. de, Cavalcanti, H. A. F., \& Lameu, J. N. (2014). Vulnerabilidade e bem-estar psicológicos do estudante universitário. Revista Brasileira de Terapia Cognitiva, 10(1), 2-10. Recuperado em 23 de março de 2017, de http://pepsic.bvsalud.org/pdf/rbtcc/v10n1/v10n1a02.pdf 
Pureza, J. R., Rusch, S. G. S., Wagner, M., \& Oliveira, M. S. (2012). Treinamento de habilidades sociais em universitários: uma proposta de intervenção. Revista Brasileira de Terapias Cognitivas, 8(1), 2-9. Recuperado em 18 de julho de 2016, de http://pepsic.bvsalud.org/scielo.php?script=sci_arttext\&pid=S1808-56872012000100002\&lng=pt\&tlng=pt

Ramos, F. P., Kuster, N. S., Lucas, J. N., Nascimento, C. P. (2019). Oficina de controle de controle de ansiedade e enfrentamento de estresse com universitários. Revista Psi UNISC, 3(1), 121-140.

Rodrigues, M. E. (2005). Estudar: como ensinar? Em: Guilhardi, H.J. \& Aguirre, N.C. de (Orgs.). Sobre Comportamento e Cognição: expondo a variabilidade (pp. 416-427). Santo André: ESETec Editores Associados.

Rogers, C. R. (2001). Tornar-se pessoa. São Paulo: Martins Fontes.

Sampaio, R. K. N., \& Bariani, I. C. D. (2011). Procrastinação acadêmica: Um estudo exploratório. Estudos Interdisciplinares em Psicologia, 2(2), 242-262. doi: 10.5433/2236-6407.2011v2n2p242

Sampaio, R. K. N., Polydoro, S. A. J., \& Rosário, P. S. L. F. (2012). Autorregulação da aprendizagem e a procrastinação acadêmica em estudantes universitários. Cadernos de Educação, 42, 119-142. Recuperado em 23 de agosto de 2016, de https://periodicos.ufpel.edu.br/ojs2/index.php/caduc/article/view/2151

Savickas, M. L., Nota, L., Rossier, J., Dauwalder, J. P., Duarte, M. E., Guichard, J., Soresi, S., Esbroeck, R. V., \& Van Vianen, A. E. M. (2009). Life designing: A paradigm for career construction in the 21st century. Journal of Vocational Behavior, 75(3), 239-250. doi: 10.1016/j.jvb.2009.04.004

Silva, É. C., \& Tucci,A. M. (2015). Intervenção breve para redução do consumo de álcool e suas consequências em estudantes universitários brasileiros. Psicologia Reflexão e Crítica, 28(4), 728-736. doi: 10.1590/1678-7153.201528410.

Silva, M. Z., \& De Andrade, A. L. (2016). Avaliando pensamentos negativos sobre a carreira: o desenvolvimento de uma medida (EPNC). Revista Brasileira de Orientação Profissional, 17(2), 175-187. Recuperado em 17 de junho de 2018, de http://pepsic.bvsalud.org/scielo.php?script=sci_arttext\&pid=S1679-33902016000200006\&lng=pt\&tlng=pt.

Silva, S. S., \& Bardagi, M. P. B. P. (2016). Intervenções de carreira no ensino superior: estrutura dos serviços na Grande Florianópolis. Revista Psicologia em Foco, 8(12), 14-32. Recuperado em 10 de Junho de 2018, de http://revistas. fw.uri.br/index.php/psicologiaemfoco/article/view/2462

Soares, A. B., Francischetto, V., Dutra, B. M., Miranda, J. M., Nogueira, C. C. C., Leme, V. R., Araújo, A. M., \& Almeida, L. S. (2014). O impacto das expectativas na adaptação acadêmica dos estudantes no Ensino Superior. Psico-USF, 19(1), 49-60. doi: 10.1590/S1413-82712014000100006

Soares, A. B., Mourão, L., Valory, t., \& Mello, S. (2011). Estudo para a construção de um instrumento de comportamentos acadêmico-sociais para estudantes universitários. Estudos e Pesquisas em Psicologia, 11(2), 488-506. doi: 10.12957/ epp. 2011.8386

Stricker, G., \& Gold, J. R. (Ed.). (2013). Comprehensive handbook of psychotherapy integration. New York: Springer.

Teixeira, M. O., \& Costa, C. J. (2017). Carreira e bem-estar subjetivo no ensino superior: Determinantes pessoais e situacionais. Revista Brasileira de Orientação Profissional, 18(1), 19-29. DOI: 10.26707/1984-7270/2017v18n1p19

Zeferino, M. T., Hamilton, H., Brands, B., Wright, M. G. M., Cumsille, F., \& Khenti, A. (2015). Consumo de drogas entre estudantes universitários: família, espiritualidade e entretenimento moderando a influência dos pares. Texto \& Contexto Enfermagem, 24, 125-135. Recuperado em 10 de abril de 2016, de http://www.redalyc.org/articulo. oa? $\mathrm{id}=71439758016$

Recebido 30/01/2018

$1^{a}$ Reformulação 03/06/2018

Aceite Final 07/10/2018

Sobre os autores

Fabiana Pinheiro Ramos é Doutora em Psicologia. Docente do Departamento de Psicologia da Universidade

Federal do Espírito Santo. Desenvolve estudos e pesquisas em clínica comportamental com diversas populações. Participa do Grupo de Pesquisa em Psicologia Pediátrica da ANPEPP e do Grupo de Pesquisa Processos Psicológicos e Saúde do CNPq.

Alexsandro Luiz De Andrade é Doutor em Psicologia. Coordenador do Laboratório de Avaliação e Mensuração Psicológica-AMP. Docente do Departamento de Psicologia e do Programa de Pós-Graduação em Psicologia da Universidade Federal do Espírito Santo. Bolsista de Produtividade CNPq.

Adriano Pereira Jardim é Doutor em Psicologia. Docente do Departamento de Psicologia e do Programa de PósGraduação em Psicologia Institucional da Universidade Federal do Espírito Santo-ES, Brasil.

Juliana Nascimento Lucas Ramalhete é Psicóloga pela Universidade Federal do Espírito Santo-ES, Brasil.

Gustavo Pfister Pirola é Psicólogo pela Universidade Federal do Espírito Santo-ES, Brasil.

Caroline Egert é Psicóloga pela Universidade Federal do Espírito Santo-ES, Brasil. 